\title{
Simulating Nonlinear Deformations of Solar Sail Membranes Using Explicit Time Integration
}

\author{
John T. Wang*, Tzikang Chen ${ }^{\dagger}$, David W. Sleight**, and Alex Tessler** \\ NASA Langley Research Center, Hampton, VA 23681-2199
}

\begin{abstract}
In this study, the explicit time integration method is employed to predict deformations of highly flexible solar sail structural components. The nonlinear static analysis of a highly flexible ribbon structure is presented to demonstrate the need for having the explicit time integration method in the analysis toolbox for solar sails. Static analyses of the ribbon structure produce ambiguous results whereas the explicit time integration method determines the correct results. Extensive benchmarking examples are also presented to build confidence in the use of the explicit method. Previously determined nonlinear wrinkling deformations of solar sail membranes are found by the explicit method. As the explicit method is known to often require more computational time than nonlinear static methods, a study on mass scaling was also conducted. The computational times are reported for the nonlinear static and explicit time integration solutions to calibrate the advantage of using mass scaling for these problems.
\end{abstract}

\section{Introduction}

Ultra-Lightweight Inflatable Structures have become very attractive because they can meet structural requirements for space applications at a low cost. Recently NASA's In-Space Propulsion (ISP) technology program has supported the development of solar sails for deep space science exploration missions. Solar sails capture the momentum of sunlight photons ${ }^{1}$ as their source of propulsion. Since the gained momentum per unit sail-surface area is quite small, solar sails must be very large, and the sail membranes must be thinner than paper to produce useful thrust for the attached spacecraft.

A typical sail is constructed from two major structural components, booms and sail membranes. ${ }^{2,3}$ In order to minimize payload packaging, the long supporting booms and sail membranes need to be folded to fit into the shroud of a small launch vehicle. The booms can be mechanically deployed as isogrid booms or inflation-deployed as thin walled tubes. The unfolding of the sail membranes can occur during or after the boom deployment. Billowed and wrinkled solar sails are less effective than flat solar sails due to the reduced momentum exchange provided by oblique incidence photons. To keep billowing within allowable limits, membrane tension is needed. However, the membrane tension may exacerbate the sail's wrinkling problem.

Quasi-static modeling methods can be used to determine the static wrinkling and billowing deformations of solar sail membranes. When nonlinear Newton-Raphson and Riks methods are employed to obtain these deformations, a near singular tangent stiffness matrix is often encountered resulting in poor convergence rates or in convergence to physically unrealistic solutions. Quasi-static modeling with explicit time integration is an alternative approach which does not require the inverse of a near singular matrix. ${ }^{4}$ The drawback to employing explicit time integration is that very small time steps are required to maintain accuracy and algorithm stability. As a result, quasi-static modeling is computationally intensive.

In this paper, an overview of the explicit time integration method is first presented. Analyses of a laterally loaded ribbon-like sail strip using a nonlinear static solver and the explicit time integration solver are then conducted. This problem demonstrates the need for using the explicit time integration method in the analysis of solar sail structures. Finally, benchmark analyses of solar sail wrinkling and billowing problems are performed, employing various loading rates and mass scaling methods to reduce the computational time of the explicit time integration method.

\footnotetext{
* Aerospace Engineer, Analytical and Computational Method Branch/Structures and Materials Competency, MS 240, AIAA Senior Member.

**Aerospace Engineer, Analytical and Computational Method Branch/Structures and Materials Competency, MS 240, AIAA Member.

${ }^{\dagger}$ Mechanical Engineer, Army Research Laboratory, Vehicle Technology Directorate, MS 240.
} 


\section{Explicit Time Integration}

The equations of motion of a discretized nonlinear structural system can be written as

$$
\boldsymbol{M u}+\boldsymbol{C u}+f_{\text {int }}=\boldsymbol{f}_{\text {ext }}
$$

where $\dot{\boldsymbol{u}}$ and $\ddot{\boldsymbol{u}}$ are the vectors of generalized velocities and accelerations, respectively. $\boldsymbol{M}$ and $\boldsymbol{C}$ are the mass and damping matrices, and $\boldsymbol{f}_{\text {int }}$ and $\boldsymbol{f}_{\text {ext }}$ are the vectors of the internal and external forces. The internal forces include the effects of material and geometric nonlinearities. Therefore, the internal force vector has to be updated at each time step during the time integration of the equations of motion. At the current configuration, the internal forces may be evaluated from ${ }^{4}$

$$
\boldsymbol{f}_{\text {int }}=\int_{V} \boldsymbol{B}^{T} \boldsymbol{\sigma} d V
$$

where $\boldsymbol{B}$ is the strain-displacement matrix, $V$ is the current volume, and $\boldsymbol{\sigma}$ is the vector of Cauchy stresses. For linear problems, the internal forces can be written as

$$
f_{\text {int }}=\boldsymbol{K u}
$$

where $\boldsymbol{K}$ is the stiffness matrix of the system, and $\boldsymbol{u}$ is the vector of generalized displacements.

The equations of motion of a discretized nonlinear structural system can be solved by an explicit time integration method. The explicit method presented in the following section uses a central difference time integration scheme with lumped mass and damping matrices. In this system of equations, the variables of the current time step form a vector on the left-hand-side, and the terms on the right-hand-side of the equation are known from the previous time step. Only simple algebraic manipulations are needed to obtain the system solutions.

\section{A. Central Difference Scheme for Explicit Time Integration}

The central difference scheme to approximate $\dot{\boldsymbol{u}}$ and $\ddot{\boldsymbol{u}}$ for integrating the equation of motion is given by

$$
\begin{aligned}
& \dot{\boldsymbol{u}}^{t}=\frac{1}{\Delta t}\left(\boldsymbol{u}^{t+\frac{1}{2} \Delta t}-\boldsymbol{u}^{t-\frac{1}{2} \Delta t}\right) \\
& \ddot{\boldsymbol{u}}^{t}=\frac{1}{\Delta t}\left(\dot{\boldsymbol{u}}^{t+\frac{1}{2} \Delta t}-\dot{\boldsymbol{u}}^{t-\frac{1}{2} \Delta t}\right)
\end{aligned}
$$

where $\Delta t$ is the time step length. Substituting Eq. (4) into Eq. (1), and employing lumped mass, $\boldsymbol{M}$, and damping $\boldsymbol{C}=\alpha \boldsymbol{M}$ matrices (where $\alpha$ is the damping ratio), the explicit solution of Eq. (1) becomes

$$
\begin{aligned}
& \dot{u}_{i}^{t+\frac{1}{2} \Delta t}=\frac{2-\alpha \Delta t}{2+\alpha \Delta t} \dot{u}_{i}^{t-\Delta t}+\frac{2 \Delta t}{m_{i i}(2+\alpha \Delta t)}\left(f_{\text {ext }_{i}}^{t}-f_{\text {int }}^{t}\right) \\
& u_{i}^{t+\Delta t}=u_{i}^{t}+\Delta t \dot{u}_{i}^{t+\frac{1}{2} \Delta t}
\end{aligned}
$$

In Eq. (5), $m_{i i}$ is the diagonal term of the mass matrix related to the degree-of-freedom $i ; f_{\text {int }}^{t}$ is the internal force related to the degree-of-freedom $i$, which is calculated using Eq. (2); and $f_{\text {ext }}^{t}$ is the given external force 
corresponding to the same degree-of-freedom $i$. Note that the terms in the right hand side of Eq. (5) are readily available.

The central difference scheme ${ }^{5}$ (with no damping) is stable for a time step increment satisfying the following inequality

$$
\Delta t \leq 2 / \omega_{\max }
$$

where $\omega_{\max }$ is the highest natural frequency of the finite element (FE) model. Damping can reduce the stable time step. An upper bound for the stable time step with damping is given by

$$
\Delta t \leq \frac{2}{\omega_{\max }}\left(\sqrt{1+\xi_{\max }^{2}}-\xi_{\max }\right)
$$

where $\xi_{\max }$ is the fraction of critical damping in the mode with the highest natural frequency.

An approximation to the maximum stable time step is often written as the smallest transit time required for a sound wave to travel through any of the elements in the mesh $^{5}$

$$
\Delta t \approx \frac{L_{\min }}{S}
$$

where $L_{\min }$ is the smallest element dimension in the mesh, and where $S$ is the speed of sound. For an isotropic shell element, the speed of sound can be expressed as ${ }^{6}$

$$
S=\sqrt{\frac{E}{\left(1-v^{2}\right) \rho}}
$$

where $E$ is the Young's modulus, $v$ is the Poisson's ratio, and $\rho$ is the material density. For shell elements, the element thickness is not considered in determining the smallest element dimension; the stability limit is based upon the midplane dimensions only.

\section{B. Quasi-static Analysis}

The quasi-static analysis approach described in ABAQUS/Explicit User's Manual ${ }^{7}$ requires that the dynamic effects induced by the applied load are small. One general means of evaluating whether or not an explicit simulation is producing an appropriate quasi-static solution relies on studying the various energies of the structural model. The two most important energies are the internal energy and the kinetic energy. For an elastically deformed structure, the internal energy is the strain energy. Dynamic relaxation (DR) methods ${ }^{8,9}$ can be used to determine a critical damping coefficient. The critical damping coefficient is the optimal value for damping. Using the critical damping coefficient enables the steady state solution to be reached in minimum computational time. Since the DR methods are not implemented into ABAQUS, the damping coefficients used in this paper were selected with a trial and error approach to assure that quasi-static solutions could be obtained. The following ad hoc rules were used to determine if a quasi-static solution had been achieved:

1. The kinetic energy of the deformed structure shall not exceed a small fraction (about 5\%) of its internal energy throughout most of time period of the explicit analysis.

2. The ratio of the kinetic energy versus the internal energy shall be less than $0.1 \%$ at the steady state.

3. The time rate of change of the internal energy shall be negligible at the steady state.

4. The maximum out-of-plane deformation shall reach a constant value at the steady state.

It often takes an intensive computational effort to obtain the quasi-static solution if the natural time scale of the structural model is used. The natural time scale is the stable time step length determined from the actual material 
properties. To obtain a computationally efficient solution, a larger time step or a faster loading rate needs to be used. The mass scaling approaches in ABAQUS ${ }^{7}$ can be used to increase the stable time step length by increasing the mass density of some or all of the elements in the FE model. A fast loading rate can also be used so that the same physical event occurs in less time. As long as the dynamic effects are insignificant, the same static solution should be obtained.

\section{Numerical Simulation of Solar Sail Deformations}

Analyses using a nonlinear static analysis solver and the explicit method were conducted to simulate the geometrically nonlinear deformations of solar sail membranes. The nonlinear static analysis of a highly flexible thin sail ribbon in a stripped sail ${ }^{2}$ is presented to demonstrate the need for using the explicit time integration method for the analysis of solar sails. The sail membrane deformations, including wrinkling induced by the in-plane tension loads and billowing induced by gravity or solar pressure load, were predicted using the quasi-static analysis approach and the explicit method implemented in the ABAQUS/Explicit code. ${ }^{7}$ The analyses are benchmarked against the results using a nonlinear static analysis solver. The ABAQUS code was selected because it has both nonlinear static and explicit analysis capabilities. Results generated by both types of analyses were compared, and the feasibility of using the explicit method to simulate sail membrane nonlinear deformations was assessed. Furthermore, the use of mass scaling techniques and accelerated loading to shorten the total computational time was investigated.

\section{A. Thin Sail Ribbon under Gravity Load}

An unstretched thin ribbon with dimensions of 100 meters long, one meter wide, and one micrometer thick as shown in Fig. 1 was analyzed. The ribbon is pinned at both ends and deforms under its own weight. The material properties are listed in Table 1.

Table 1: Kapton properties

\begin{tabular}{|l|l|}
\hline Thickness & $1.0 \times 10^{-6} \mathrm{~m}$ \\
\hline Modulus & $2.6 \times 10^{9} \mathrm{~N} / \mathrm{m}^{2}$ \\
\hline Poisson's Ratio & 0.3 \\
\hline Density & $1420 \mathrm{~kg} / \mathrm{m}^{3}$ \\
\hline
\end{tabular}

The exact solution of the maximum deflection at the mid-span, $W_{\max }$, is given by the expression ${ }^{10}$

$$
W_{\max }=-\sqrt[3]{\frac{3 \rho g L^{4}}{64 E}}
$$

where $L$ is the length of the ribbon, $\rho$ is the density, and $g=9.8 \mathrm{~m} / \mathrm{s}^{2}$ is the acceleration due to gravity. For the given ribbon, the maximum deflection is $-2.927 \mathrm{~m}$.

Nonlinear static analyses were performed using four different types of elements in the ABAQUS code ${ }^{11}$ (the M3D4 membrane element and the S4, S4R, and S4R5 shell elements). The S4 element is a full integration shell element, and the S4R and S4R5 elements are reduced integration elements. Two different FE meshes were used: (1) a coarse mesh consisting of 100 elements and (2) a fine mesh consisting of 500 elements. Each model has four elements in the width direction. Initially, the nonlinear static analyses cannot predict transverse displacements because the ribbon has no stiffness associated with bending. In mathematical terms, the initial stiffness matrix is nearly singular or highly ill-conditioned. Two techniques were used to circumvent the matrix singularity problem. One technique is to introduce a membrane tension by slightly reducing the temperature in the membrane. The coefficient of thermal expansion (CTE) is assumed to be $1.6 \times 10^{-5} /{ }^{\circ} \mathrm{C}$. The pre-tension and gravitational loads were applied in three separate load steps. In the first load step, a temperature of $-10^{\circ} \mathrm{C}$ was applied to all nodes in the model to pre-tension the ribbon. In the second load step, the temperature was held constant; and the gravitational loading was applied. Finally, in the third load step, the temperature load was removed. The other technique is to use an ABAQUS automatic mechanism for stabilizing the nonlinear solution in which a volume-proportional damping force is automatically added to the model to overcome convergence difficulties. This mechanism is triggered by including the STABILIZE parameter in the ABAQUS nonlinear static solution procedure. 
The nonlinear static analysis results for the thin ribbon are summarized in Table 2 . The analysis results of the coarse mesh model with the M3D4 membrane element converged if one of the two singularity relieving techniques was used. The deformed shapes for all analyses using the M3D4 elements are similar to the deformed shape shown in Fig. 2(a) for the coarse mesh model. The analysis results of the refined mesh model with the stabilizing techniques also converged for the M3D4 element. However, the maximum deflections are significantly smaller than the exact solution. The deformed shape for the refined mesh model shown in Fig. 2(b) is quite different from the deformed mesh for the coarse mesh model shown in Fig. 2(a).

Table 2: Results for $100 \mathrm{~m}$ thin ribbon

\begin{tabular}{|c|c|c|c|c|c|c|}
\hline $\begin{array}{l}\text { Analysis } \\
\text { Type }\end{array}$ & $\begin{array}{l}\text { Element } \\
\text { Type }\end{array}$ & $\begin{array}{l}\text { Mesh } \\
\text { (Elements) }\end{array}$ & Pretension & $\begin{array}{l}\text { STABLIZE } \\
\text { Parameter }\end{array}$ & $\begin{array}{l}\text { Converged } \\
\text { Solution }\end{array}$ & $\begin{array}{l}\text { Max Deflection } \\
\text { (m) }\end{array}$ \\
\hline \begin{tabular}{|l|} 
Exact \\
Solution
\end{tabular} & & & & & N/A & $-2.927 * *$ \\
\hline \multirow{23}{*}{$\begin{array}{l}\text { Nonlinear } \\
\text { Static } \\
\text { Analysis }\end{array}$} & \multirow[t]{7}{*}{ M3D4 } & $100 x 4$ & Yes & & Yes & $-2.933^{* * *}$ \\
\hline & & $100 \times 4$ & No & No & No & \\
\hline & & $100 \times 4$ & & 0.0002 & Yes & -2.917 \\
\hline & & $100 \times 4$ & & 0.004 & Yes & -2.921 \\
\hline & & $500 \times 4$ & Yes & & Yes & $-2.933^{* * *}$ \\
\hline & & $500 \times 4$ & & 0.0002 & Yes & $-0.7680^{\#}$ \\
\hline & & $500 \times 4$ & & 0.004 & Yes & -0.4066 \\
\hline & \multirow[t]{5}{*}{ S4 } & $100 \times 4$ & Yes & & No & \\
\hline & & $100 \times 4$ & & 0.0002 & No & \\
\hline & & $100 x 4$ & & 0.001 & No & \\
\hline & & $500 \times 4$ & & 0.0002 & No & \\
\hline & & $500 \times 4$ & & 0.0004 & No & \\
\hline & \multirow[t]{5}{*}{ S4R } & $100 x 4$ & Yes & & No & \\
\hline & & $100 \times 4$ & & 0.0004 & No & \\
\hline & & $100 \times 4$ & & 0.001 & No & \\
\hline & & $100 \times 4$ & & 0.01 & No & \\
\hline & & $500 \times 4$ & & 0.0002 & No & \\
\hline & \multirow[t]{6}{*}{ S4R5 } & $100 \times 4$ & Yes & & No & \\
\hline & & $100 \times 4$ & & 0.0002 & Yes & -2.102 \\
\hline & & $100 \times 4$ & & 0.0004 & Yes & -2.722 \\
\hline & & $100 \times 4$ & & 0.001 & Yes & -2.78 \\
\hline & & $500 \times 4$ & & 0.0002 & Yes & $-5.6237 \mathrm{E}-3^{\# \#}$ \\
\hline & & $500 \times 4$ & & 0.0004 & Yes & $-9.3065 \mathrm{E}-3$ \\
\hline \multirow{2}{*}{$\begin{array}{l}\text { Explicit } \\
\text { Analysis }\end{array}$} & \multirow[t]{2}{*}{ S4R } & $100 \times 4$ & No & No & N/A & $-2.933^{* * *}$ \\
\hline & & $500 \times 4$ & No & No & N/A & $-2.932^{* *}$ \\
\hline
\end{tabular}

* ABAQUS default value: 0.0002

** $\quad$ Ref. 10, Page 171

*** $\quad$ Deflection Shape shown in Fig. 2(a)

\# Deflection shape shown in Fig. 2(b)

\#\# Deflection shape in Fig. 2(c)

Using the full integration S4 element or the reduced integration S4R element for both the coarse and refined mesh models, all nonlinear static analysis results failed to converge. Using the reduced integration S4R5 element, the analysis results of the coarse mesh model with various values of the STABILIZE parameter converged and the deformed shapes were similar to that shown in Fig. 2(a). However, the maximum deflections are much less than the exact solution. Using the reduced integration S4R5 element for the refined mesh model, the analysis results with two different STABILIZE parameter values converged. However, the maximum deflections are very small and the deformed shape plotted in Fig. 2(c) is quite unexpected. The elements at both ends of the ribbon are stretched downward, whereas the interior elements all have the same downward out-of-plane deformations. From the experience of analyzing this thin ribbon problem, we found that nonlinear static analyses of thin membrane structures could encounter difficulties in getting converged solutions or producing correct results. Other methods 
such as the explicit time integration method need to be explored and used when the nonlinear static solution fails to converge.

In the explicit analysis, the reduced integration S4R shell elements were used for both the coarse and refined mesh models. In this analysis, a damping ratio, $\alpha=2.0 \mathrm{~s}^{-1}$ was used to prevent load-induced membrane out-of-plane oscillations. The full gravity load of one $g$ was applied (using a smooth ramp function) to the ribbon in 5 seconds. The full gravity load was held for another 25 seconds while the deformation reached a steady state. The maximum deflection predicted at the mid-point of the ribbon with the coarse and refined mesh models were 2.933 and 2.932 meters, respectively. Both are very close to the exact solution and the maximum deformations predicted using the M3D4 membrane element with membrane pre-tension.

\section{B. Wrinkling of Sail Membranes under Tension Loads}

Geometrically nonlinear static and explicit time integration analyses were performed to simulate the wrinkling deformations of a square $0.5 \mathrm{~m}$ solar sail membrane due to a tension load of $2.45 \mathrm{~N}$ applied at each corner. The thickness of the sail membrane is $2.54 \times 10^{-5} \mathrm{~m}$. The material properties are given in Table 1 . These analyses were performed on a desktop PC with a $2.66 \mathrm{GHZ}$ Pentium 4 processor and $768 \mathrm{MB}$ RAM. The FE model ${ }^{12}$ shown in Fig. 3 was used for the nonlinear static analysis. This full sail membrane model contains 4,640 S4R5 shell elements. Randomly generated out-of-plane imperfections, with amplitudes from $-10 \%$ to $10 \%$ of the sail thickness, were imposed on the nodes in the loaded corner regions. Detailed geometrically nonlinear static analysis procedures for using the "stabilization" parameter in simulating the wrinkling deformations of this sail membrane can be found in Ref. 12. The wrinkling deformations of this sail membrane model predicted by the nonlinear static analysis are shown in Fig. 4.

In the explicit analyses, the upper right quadrant of the full model with symmetry boundary conditions shown in Fig. 5 was used. This upper right quadrant model contains 1,160 S4R shell elements. Wrinkling deformations of the sail membrane were predicted with a damping parameter $(\alpha)$ of $0.001 \mathrm{~s}^{-1}$. The effects of loading rates and mass scaling factors on the wrinkling deformations were studied. Two loading rates were used to smoothly apply the corner loads to the membrane in $5.0 \mathrm{~s}$ and $0.05 \mathrm{~s}$, respectively. The actual mass of the membrane was used in the loading rate study. In the mass scaling study, two techniques in the ABAQUS code were applied to scale up the mass. One technique is to scale up the membrane density manually, and the other technique is to let ABAQUS automatically scale up the membrane density such that the stable time step length equals a given value.

The wrinkling deformations predicted by the explicit analyses for the quadrant model with the two loading rates are shown in Figs. 6(a) and 7(a). Note that deformations along the free edge are larger than indicated by the color contour plots. The scale of the contour plots was selected to emphasize the wrinkling deformations away from the free edges. The minimum stable time step $(\Delta t)$ for these two loading rate analyses was $1.29 \times 10^{-7}$ seconds. The internal and kinetic energies of the model as a function of time are also plotted in Figs. 6(b) and 7(b). The wrinkle patterns and the maximum out-of plane-deformation of the dominant wrinkle (maximum positive value of the legend), shown in Figs. 6(a) and 7(a), are almost the same for both loading cases. Furthermore, the kinetic energies for both loading rates are insignificant (near zero) compared to the internal energies. The results in Figs. 6 and 7 indicate that a wide range of loading rates with the explicit analysis may be used for predicting wrinkling deformations. Increasing the loading rate can significantly reduce the central processing unit (CPU) time. Obtaining the results in Fig. 7 required two CPU hours, while obtaining the results in Fig. 6 required more than two CPU weeks. The wrinkling deformations, shown in Figs. 6(a) and 7(a), are qualitatively similar to those shown in Fig. 4. However, the maximum wrinkle amplitudes (maximum positive w-displacements) shown in Figs. 6(a) and 7(a) are about $20 \%$ less than those shown in Fig. 4. The nonlinear static analysis was completed in about fourteen CPU minutes. It is apparent that the explicit analysis is very computationally intensive. In the next few paragraphs, attempts of using mass scaling to increase the stable time step length for shortening the computational time are discussed.

To shorten the total computational time, a mass scaling technique was used to uniformly scale up the membrane density by 1,000 times. The minimum stable time step $(\Delta t)$ for this case is increased to $4.1 \times 10^{-6}$ seconds. The wrinkling deformations, shown in Fig. 8(a), are almost the same as those shown in Figs. 6(a) and 7(a). The internal and kinetic energies of the model as a function of time are also plotted in Fig. 8(b). Obtaining the results shown in Fig. 8 required about nine CPU hours. An analysis with a mass scaling factor of one million times the membrane density was also attempted, and the results are very similar to those shown in Fig. 8. However, the CPU time needed for this new analysis was reduced to less than an hour. We conclude that the computational time for predicting the membrane wrinkling deformations can be significantly reduced by using the mass scaling technique. 
The use of mass scaling needs to be exercised with caution. The following case reveals that excessive mass scaling can result in inaccurate solutions. In this case, the mass scaling technique was used to increase the stable time step length, $\Delta t$, for the model to a given value of 0.001 seconds. The ABAQUS/Explicit code scales up the density of the model about sixty million times such that the minimum stable time step length of the model becomes 0.001 seconds. As shown in Fig. 9(a), the wrinkle pattern is not correctly predicted. Furthermore, both the internal and kinetic energy have an oscillating behavior as depicted in Fig. 9(b). The internal energy change is still significant at steady state. Thus, the quasi-static solution was not reached. Note that the ad hoc rules listed previously are violated.

\section{Deformation of Sail Membrane under Distributed Loads}

Explicit and nonlinear static analyses were performed to simulate the out-of-plane deformations of a solar sail quadrant subjected to distributed loads including gravity and solar pressure loadings. The analyses were performed on a single $400 \mathrm{MHz}$ processor of an SGI Octane/SE 2-400 R12000 machine with 1 GB RAM. The sail quadrant is an isosceles right triangle with a hypotenuse of $100 \mathrm{~m}$. Each corner of the sail is attached to a short $0.0005 \mathrm{~m}$ radius cable with a length of $0.714 \mathrm{~m}$. The other end of each cable is attached to a sail boom and is assumed to have fixed boundary conditions. The material properties of the sail and cables are listed in Tables 3 and 4, respectively.

Table 3: Kapton sail material properties

\begin{tabular}{|l|l|}
\hline Thickness & $1.0 \times 10^{-6} \mathrm{~m}$ \\
\hline Modulus & $2.48 \times 10^{9} \mathrm{~N} / \mathrm{m}^{2}$ \\
\hline Poisson's Ratio & 0.34 \\
\hline Density & $1425.2 \mathrm{~kg} / \mathrm{m}^{3}$ \\
\hline
\end{tabular}

Table 4: Kevlar cable properties

\begin{tabular}{|l|l|}
\hline Radius & $0.0005 \mathrm{~m}$ \\
\hline Modulus & $62.0 \times 10^{9} \mathrm{~N} / \mathrm{m}^{2}$ \\
\hline Poisson's Ratio & 0.036 \\
\hline Density & $1440 \mathrm{~kg} / \mathrm{m}^{3}$ \\
\hline CTE & $4.6 \times 10^{-6} /{ }^{\circ} \mathrm{C}$ \\
\hline
\end{tabular}

The FE model and dimensions of the solar sail quadrant are shown in Fig. 10. This model contains 4,559 S4 or S4R shell elements, $98 \mathrm{~S} 3$ shell elements, and three B31 beam elements. Note that in the nonlinear static analysis, S4 elements were used. S4R elements were used for the explicit analyses. In the nonlinear static analyses, the STABLIZE parameter value used was 0.0002 . The nonlinear static analyses were performed in two steps. The first step pre-stressed the sail quadrant by applying a temperature change of $-304.5^{\circ} \mathrm{C}$ to contract the three Kevlar cables, and the second step applied the distributed load for either gravity or solar pressure while the pre-stress was gradually removed. The CPU time for completing the nonlinear static analysis for either the gravity or solar pressure load is less than thirty minutes.

In the case of using the explicit method to predict the deformation due to the gravity load, the actual sail material density and a mass proportional damping parameter $(\alpha)$ of $10.0 \mathrm{sec}^{-1}$ were used. The damping was applied to prevent membrane trembling. The full gravity load, an acceleration of $9.8 \mathrm{~m} / \mathrm{sec}^{2}$, was applied to the membrane in 5.0 seconds. The deformed shapes of the triangular quadrant under gravity load predicted by the explicit and the nonlinear static analyses are shown in Figs. 11(a) and 11(b), respectively. Both analyses predicted the same deformed shape and magnitude of deformations. The time history of the maximum deflection, located at node A (see Fig. 10), is plotted in Fig. 12. The deflection reaches a constant value, the same as that predicted by the nonlinear static analysis, in about 15 seconds with a CPU time of about ten hours. The lengthy CPU time was caused by the short cable elements that limit the $\Delta \mathrm{t}$ to be less than $4.75 \times 10^{-5}$ seconds.

In the case of using the explicit method to predict the sail deformation due to a solar pressure load, the actual sail material density and a mass proportional damping parameter $(\alpha)$ of $1.0 \mathrm{~s}^{-1}$ were used. The solar pressure load is $9.0 \times 10^{-6} \mathrm{~N} / \mathrm{m}^{2}$ at one astronomical unit (AU), and was applied to the membrane in 10.0 seconds. The deformed shapes, not magnified for comparison with the deformed shapes shown in Fig. 11, of the triangular quadrant under the solar pressure load predicted by the explicit and the nonlinear static analyses are shown in Figs. 13(a) and 13(b), respectively. Both analyses predicted the same deformed shape and magnitude of deformations. The time history of the maximum deflection, at node A (see Fig. 10), is plotted in Fig. 14. It takes about $150.0 \mathrm{~s}$ for the maximum deflection to reach a constant value, within 1.5 percent of that predicted by the nonlinear static analysis. The CPU time used was more than ninety hours. Again the large CPU time is attributed to the short cable elements and the small solar pressure applied which results in the membrane deforming very slowly. 


\section{Concluding Remarks}

The deformation of a long thin ribbon structure laterally loaded by gravity was determined using nonlinear static and quasi-static modeling methods. As a result of performing extensive numerical studies, it was determined that the quasi-static method, which employs explicit time integration, was found to be somewhat more robust for determining the correct deformed shape than the nonlinear static method. As ribbon structures are very much like solar sail structures, the quasi-static modeling method was put to the task of determining known solutions to the wrinkling of solar sail membranes. Explicit integration is computationally intensive, so both rate of loading and mass scaling approaches were employed to investigate how to reduce the computational effort.

In summary, this study determined that quasi-static modeling is a useful alternative tool for determining the deformations of solar sail structures, that employing accelerated loading and mass scaling approaches can reduce the computational effort required by the quasi-static models, and that excessive mass scaling can destroy the accuracy of the solution when predicting wrinkling patterns.

\section{References}

${ }^{1}$ McInnes, C. R., Solar Sailing Technology, Dynamics and Mission Applications, ISBN 1-85233-102-X, Praxis Publishing Ltd, Chichester, UK, 1999.

${ }^{2}$ Greschik, G. and Mikulas, M., "Design Study of a Square Solar Sail Architecture," AIAA Paper 2001-1259, $2^{\text {nd }}$ Gossamer Spacecraft Forum, $42^{\text {nd }}$ AIAA/ASME/ASCE/AHS/ASC Structures, Structural Dynamics, and Materials Conference, Seattle, WA, 16-19 April 2001.

${ }^{3}$ Murphy, D. M. and Murphey, T. W., "Scalable Solar Sail Subsystem Design Considerations," AIAA 2002-1703, 43 AIAA/ASME/ASCE/AHS/ASC Structures, Structural Dynamics, and Materials Conference, Denver, Colorado, 22-25 April 2002.

${ }^{4}$ Zienkiewicz, O. C. and Taylor, R. L., The Finite Element Method, Fourth Edition, Volume 2, ISBN 0-07-084175-6(v.2), McGraw-Hill Book Company (UK) Limited, 1991.

${ }^{5}$ Cook, R. D., Malkus, D. S., and Plesha, M. E., Concepts and Applications of Finite Element Analysis, Third Edition, ISBN 0-471-84788-7, John Wiley \&Sons, Inc, 1989.

${ }^{6} L S-D Y N A$, Theoretical Manual, Compiled by Hallquist, J. O., Livermore Software Technology Corporation, May 1998.

${ }^{7}$ ABAQUS/Explicit User's Manual, Version 6.3, Hibbit, Karlsson \& Sorensen, Inc., Rhode Island (USA), 2002.

${ }^{8}$ Underwood, P. G., "Dynamic Relaxation,” Computational Methods for Transient Analysis, Vol. 1, 1983, pp. $245-265$.

${ }^{9}$ Papadrakakis, M., "A Method for the Automatic Evaluation of the Dynamic Relaxation Parameters," Computer Methods in Applied Mechanics and Engineering, Vol. 25, 1981, pp. 35-48.

${ }^{10}$ Roark, R. J. and Young, W. C., Formulas for Stress and Strain, ISBN 0-07-053031-9, McGraw-Hill Book Company Limited, 1975.

${ }^{11}$ ABAQUS/Standard User's Manual, Version 6.3, Hibbit, Karlsson \& Sorensen, Inc., Rhode Island (USA), 2002.

${ }^{12}$ Tessler, A., Sleight, D. W., and Wang, J. T., "Nonlinear Shell Modeling of Ultra-Thin Membranes with Emphasis on Structural Wrinkling," AIAA 2003-1931, 44 ${ }^{\text {th }}$ AIAA/ASME/ASCE/AHS/ASC Structures, Structural Dynamics, and Materials Conference, Norfolk, Virginia, 7-11 April 2003. 


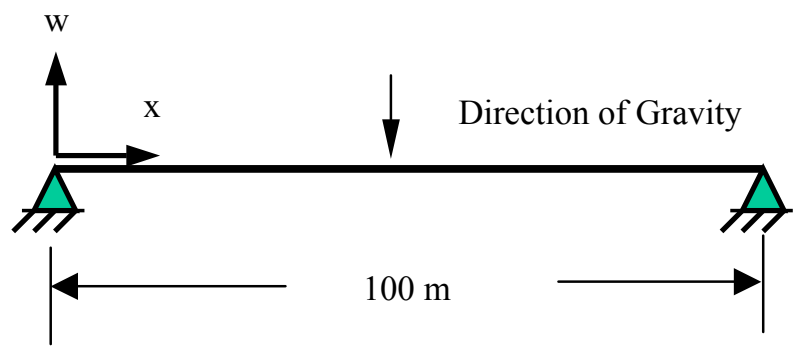

Figure 1: Kapton ribbon under gravity load; dimensions: 100 m x 1 m x $1 \mu$ m.

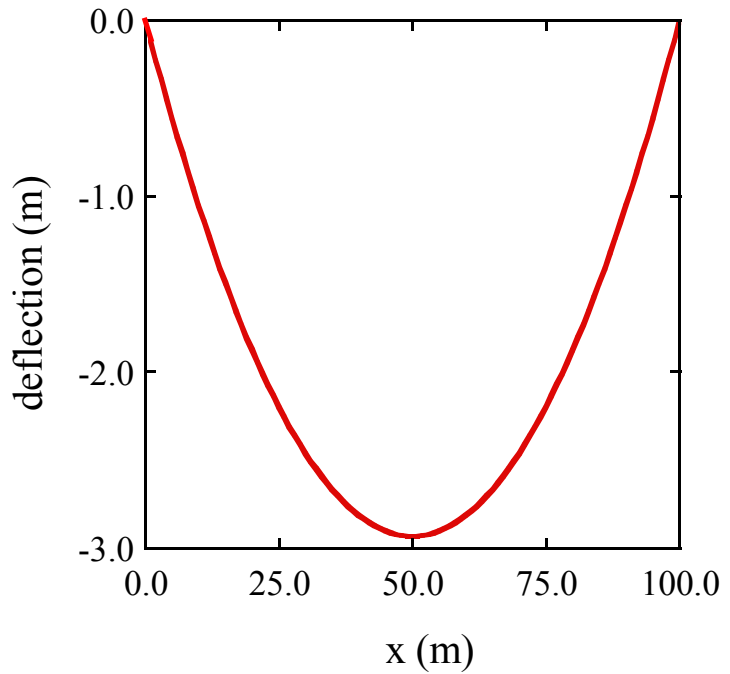

(a) M3D4 elements, 100x4 mesh, and pretension load

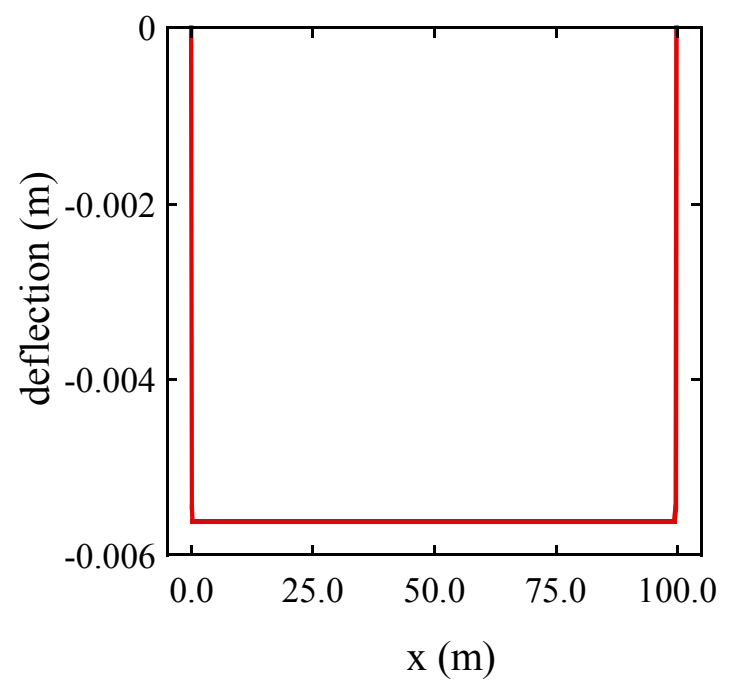

(c) S4R5 elements, 500x4 mesh, and STABLIZE parameter 0.0002

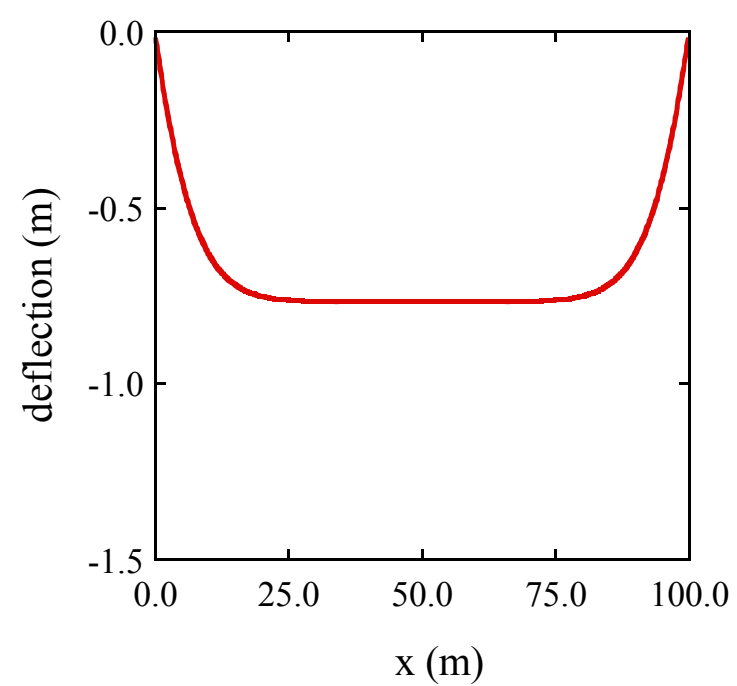

(b) M3D4 elements, 500x4 mesh, and STABLIZE parameter of 0.0002

Figure 2: Deflection shape along axial coordinate, $x$, depending on element type, mesh size, and value of the STABLIZE parameter, or pretension load used in the nonlinear static analyses. 


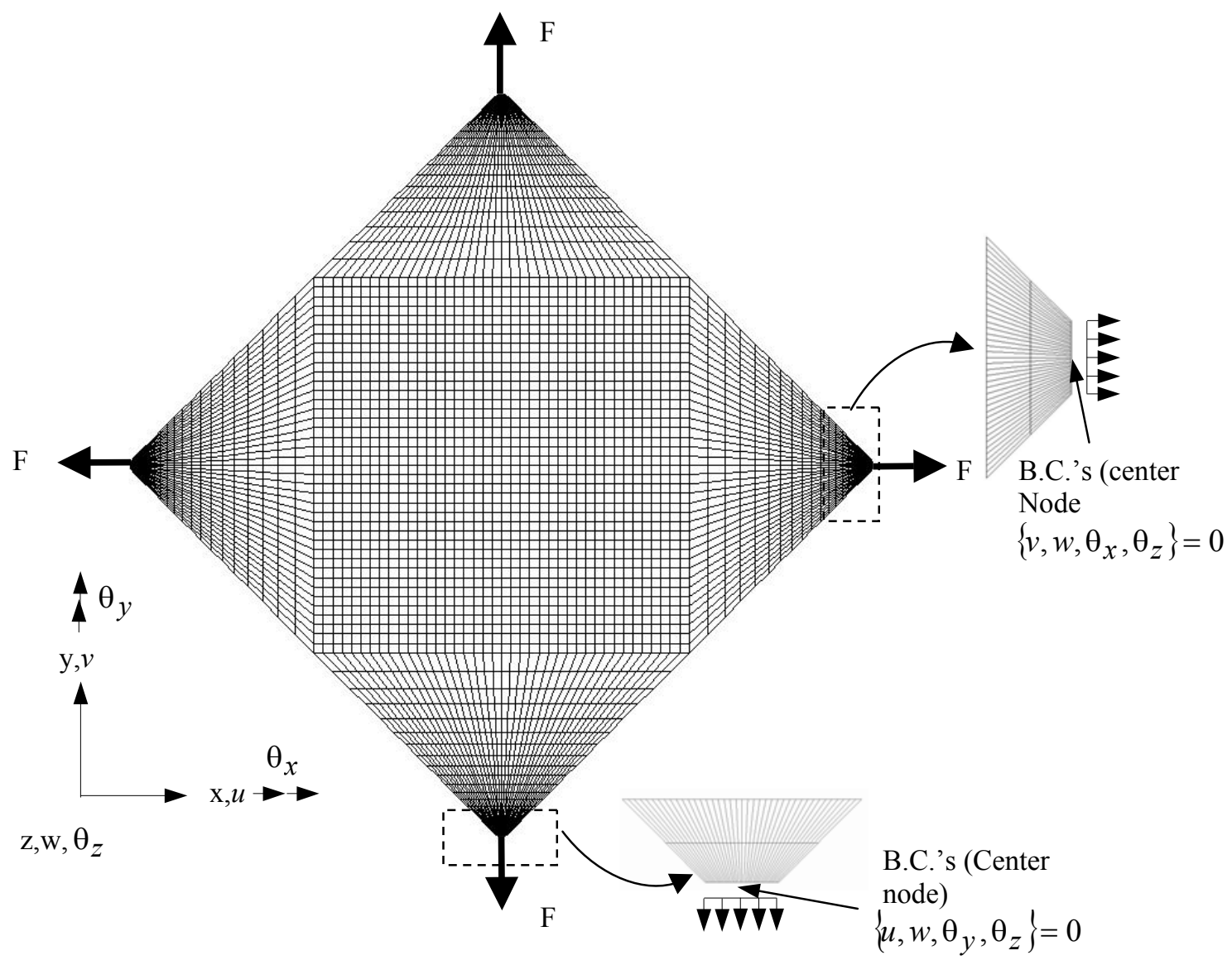

Figure 3: Finite element model of a square thin membrane $(0.5 \mathrm{~m} \times 0.5 \mathrm{~m})$ loaded in tension. ${ }^{12}$

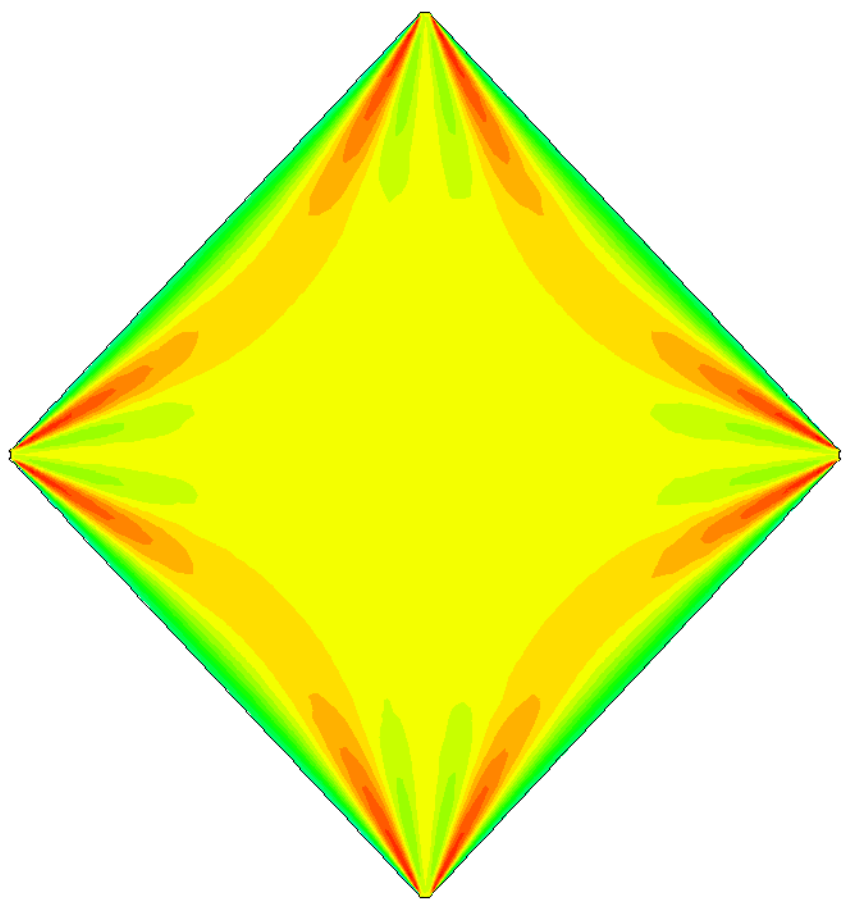

$\mathrm{w}(\mathrm{mm})$

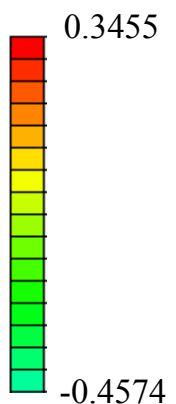

Figure 4: Wrinkling deformations of the square membrane predicted by nonlinear static analysis. 


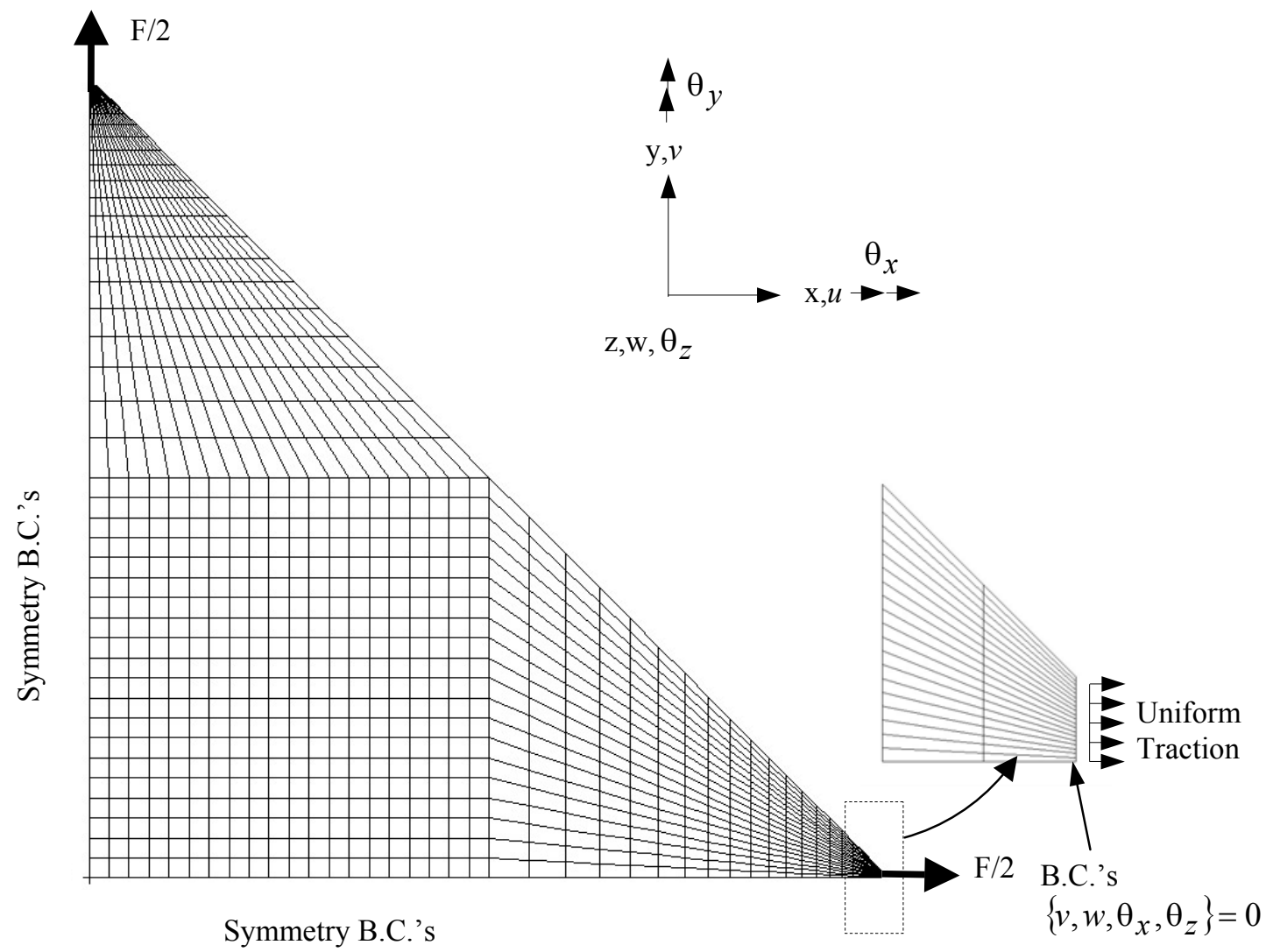

Figure 5: Symmetric upper right quadrant model for explicit analysis.

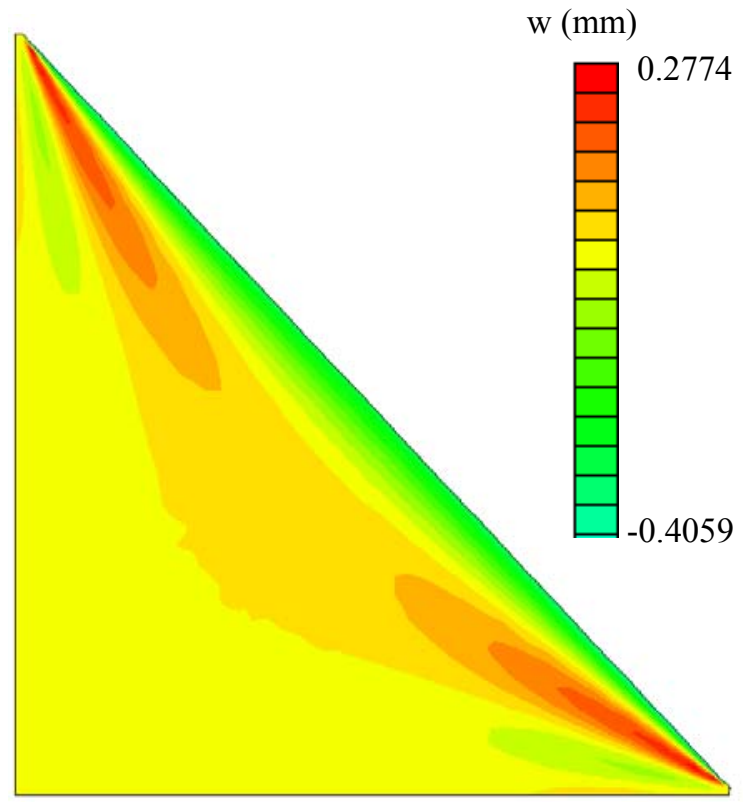

(a) Out-of-plane deformation

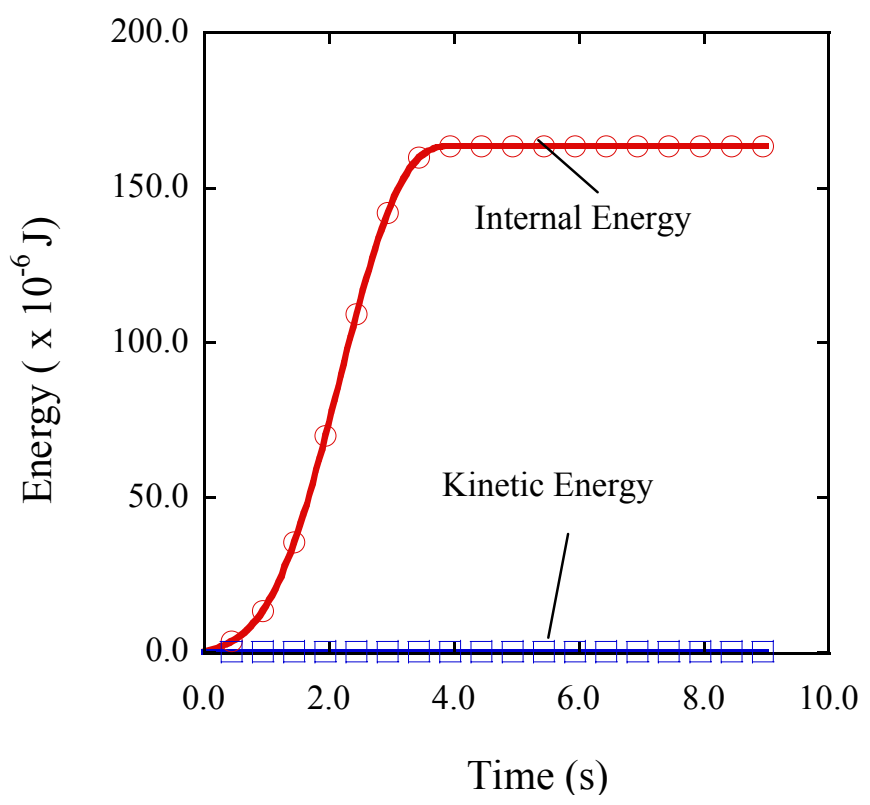

(b) Internal and kinetic energies

Figure 6: Explicit analysis results by using actual material density and taking five seconds to smoothly apply the corner loads. 


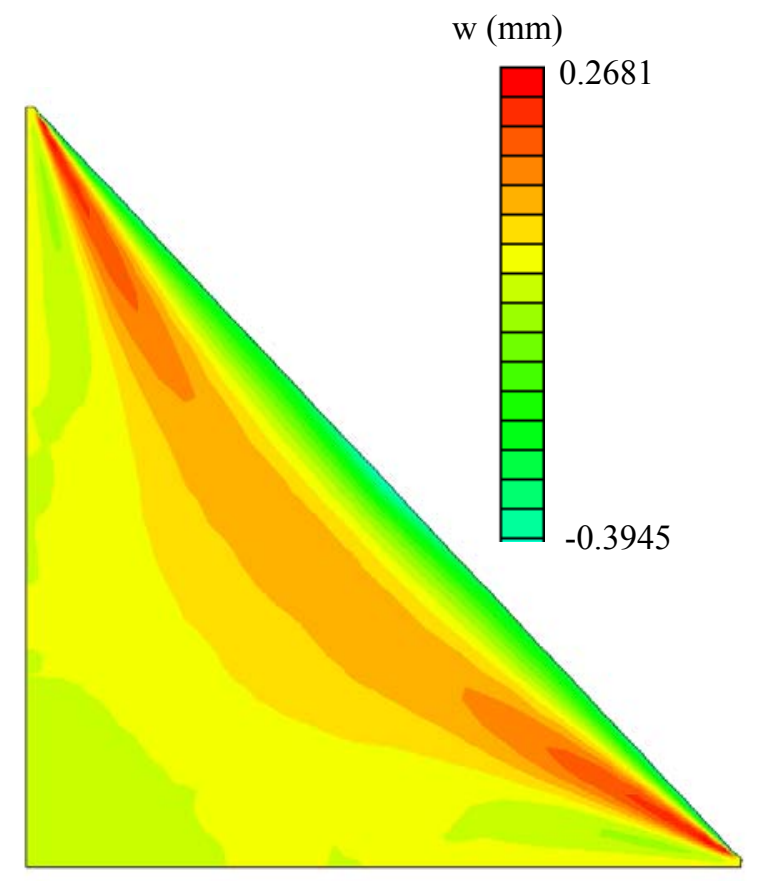

(a) Out-of-plane deformation

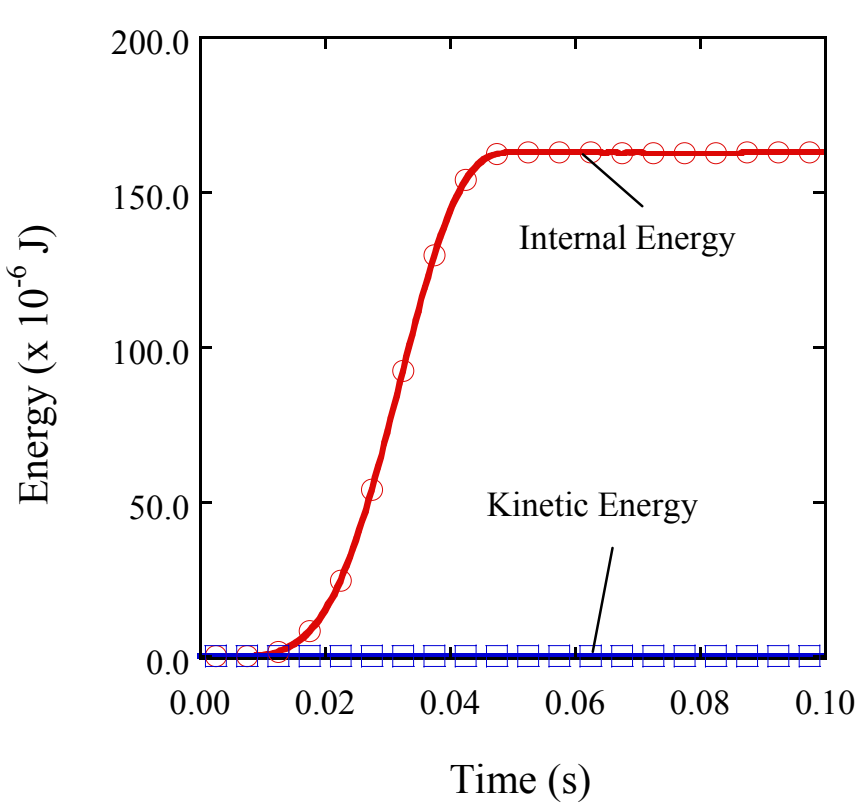

(b) Internal and kinetic energies

Figure 7: Explicit analysis results by using actual material density and taking 0.05 seconds to smoothly apply the corner loads.

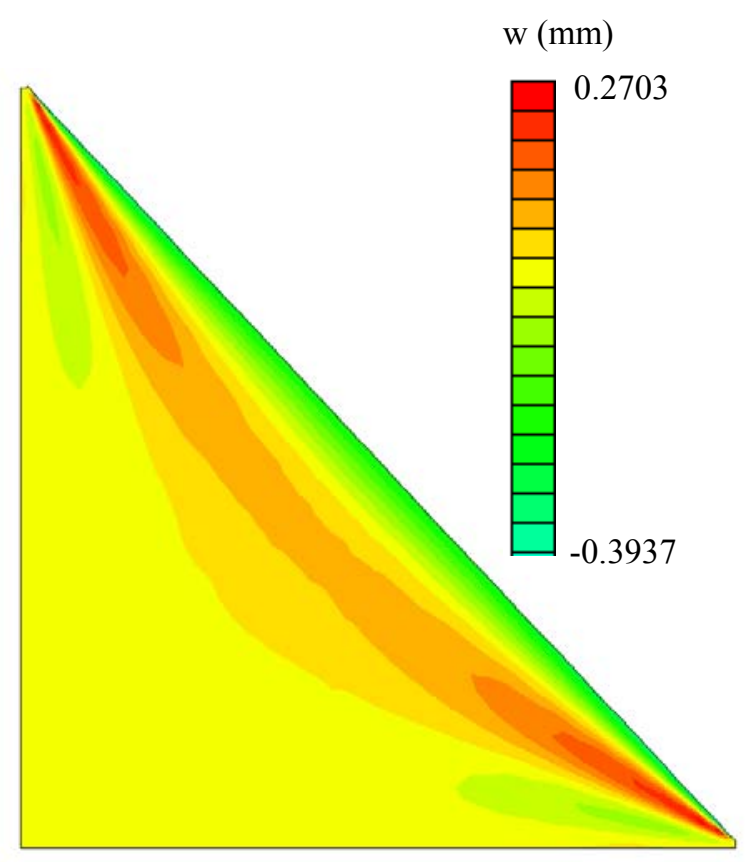

(a) Out-of-plane deformation

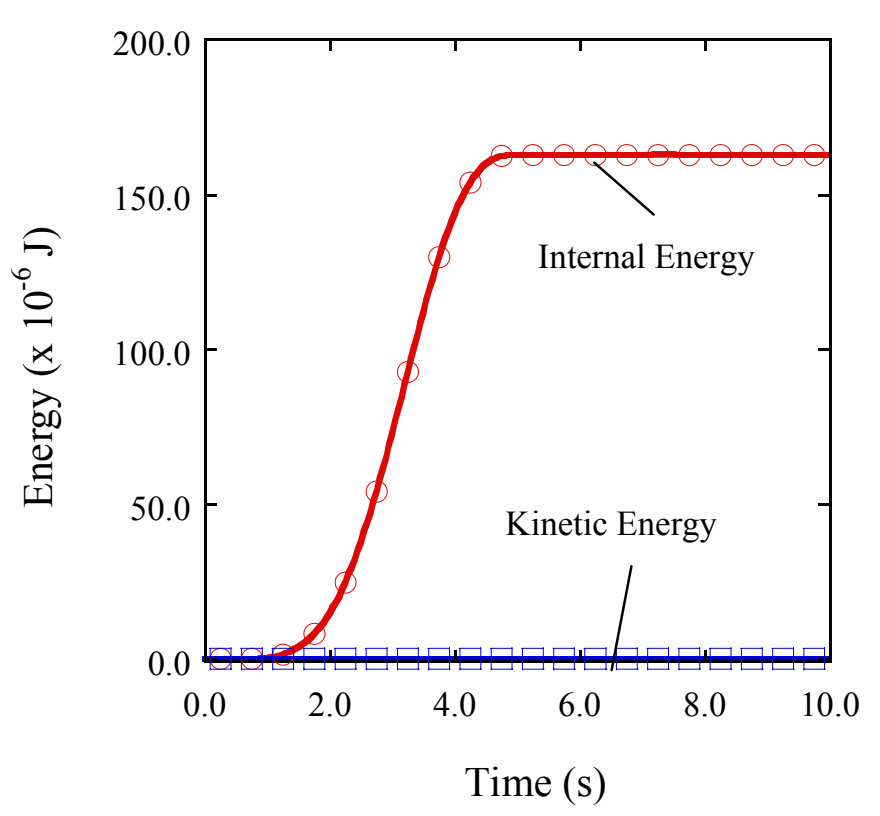

(b) Internal and kinetic energies

Figure 8: Explicit analysis results by using a uniform mass scaling of 1,000 times and taking five seconds to smoothly apply the corner loads. 


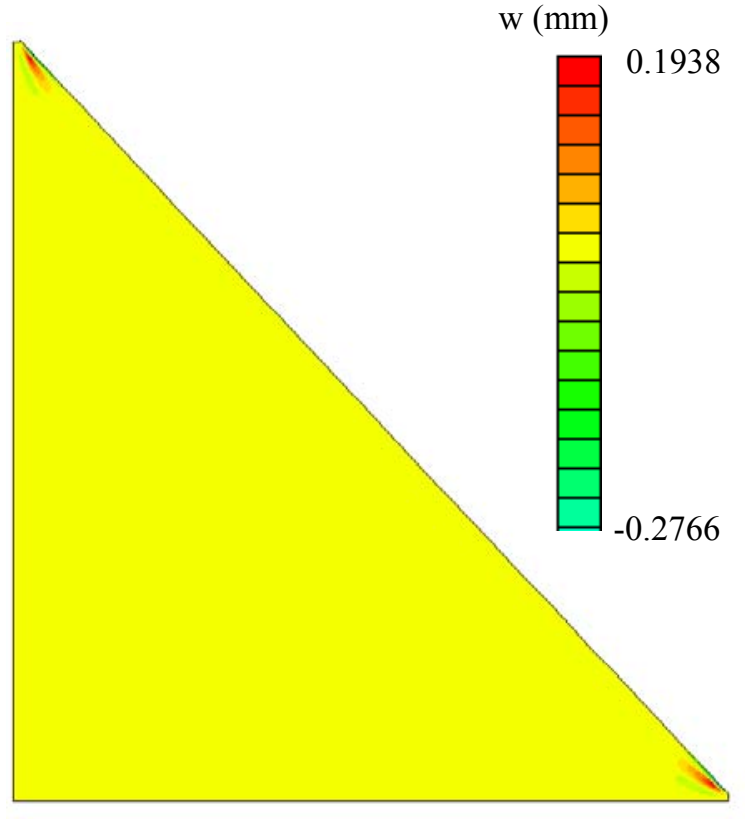

(a) Out-of-plane deformation

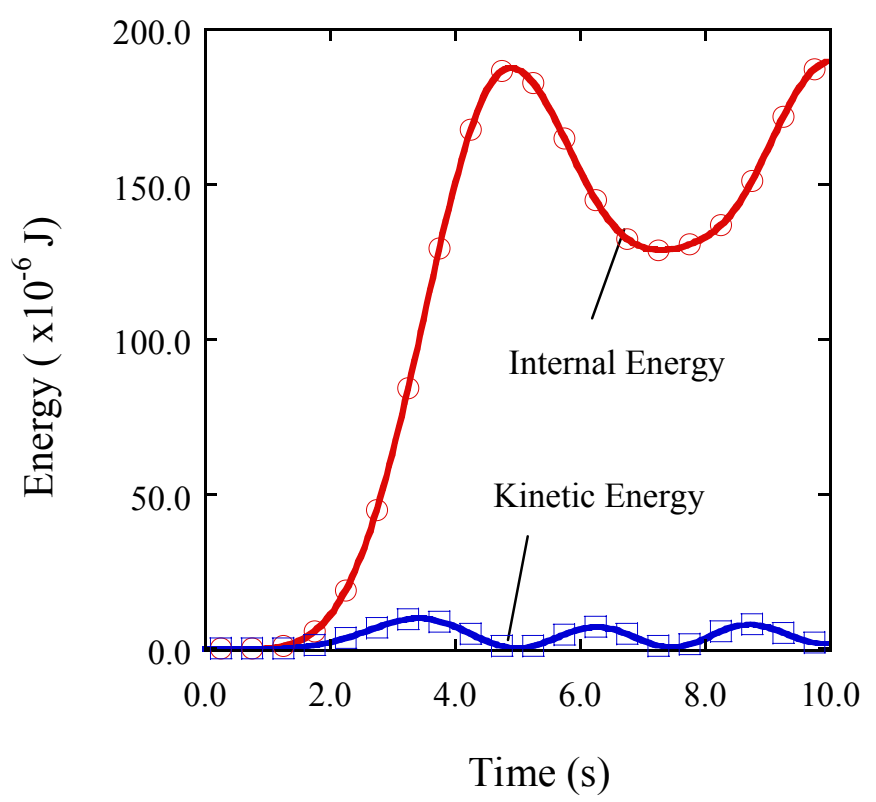

(b) Internal and kinetic energies

Figure 9: Results of an explicit analysis using a time step length of 0.001 seconds and taking 5.0 seconds to smoothly apply the corner loads.

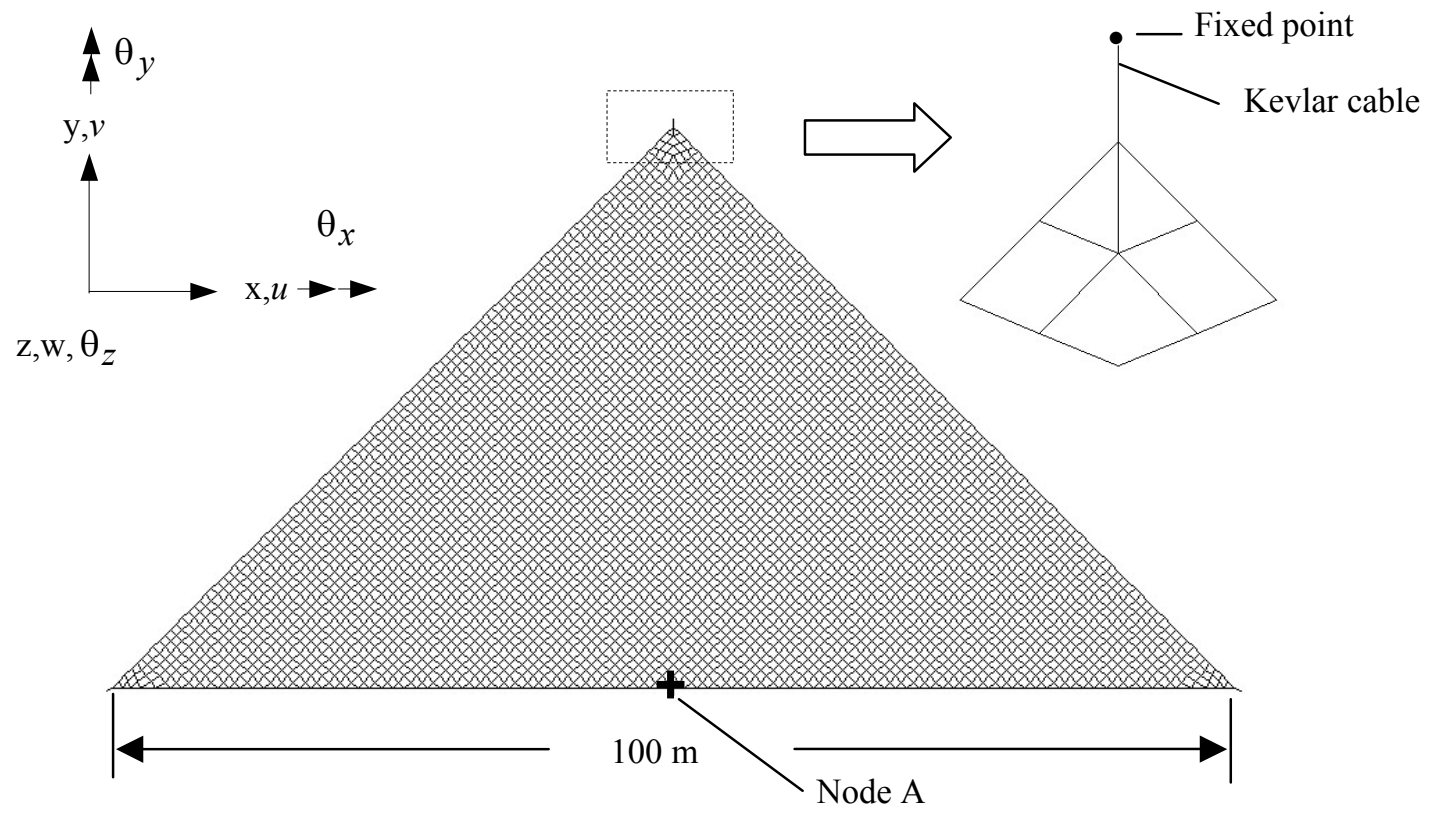

Figure 10: One quadrant of solar sail membrane with cables. 


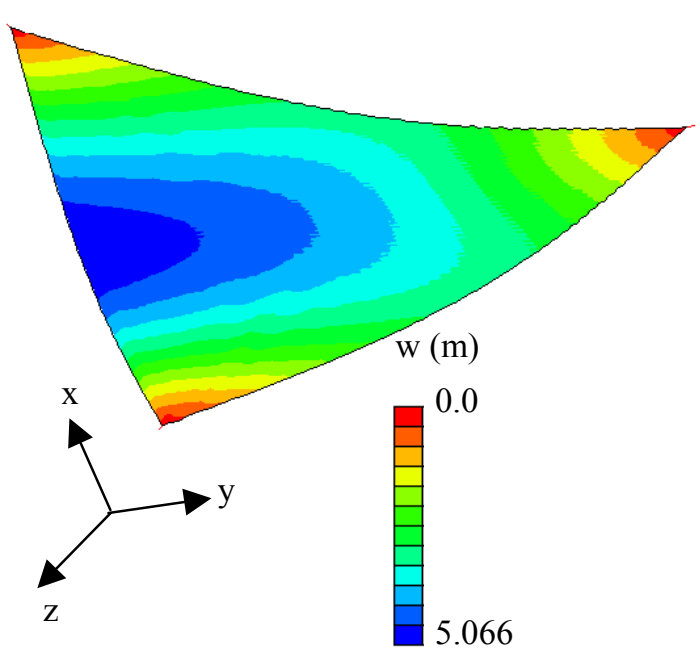

(a) Explicit analysis

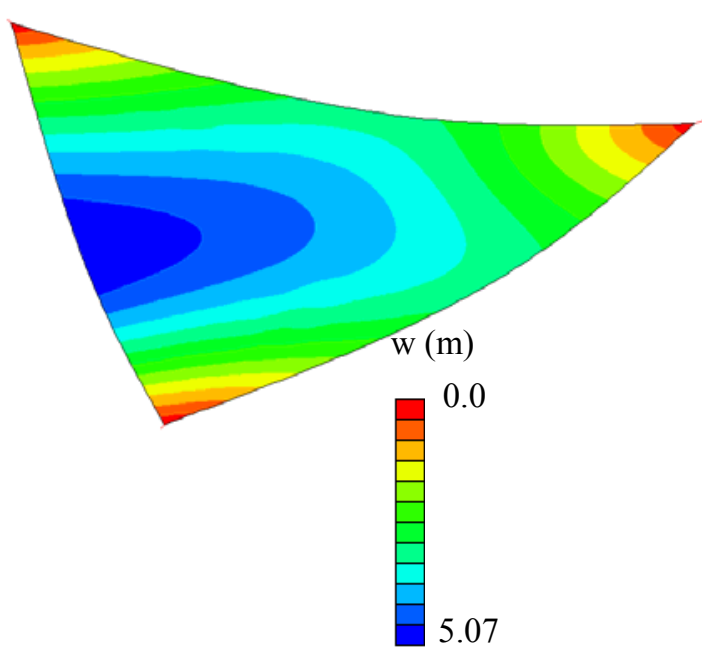

(b) Nonlinear static analysis

Figure 11: Transverse deflection of one membrane quadrant (with attached cables) of a $100 \mathrm{~m}$ solar sail due to the gravity load.

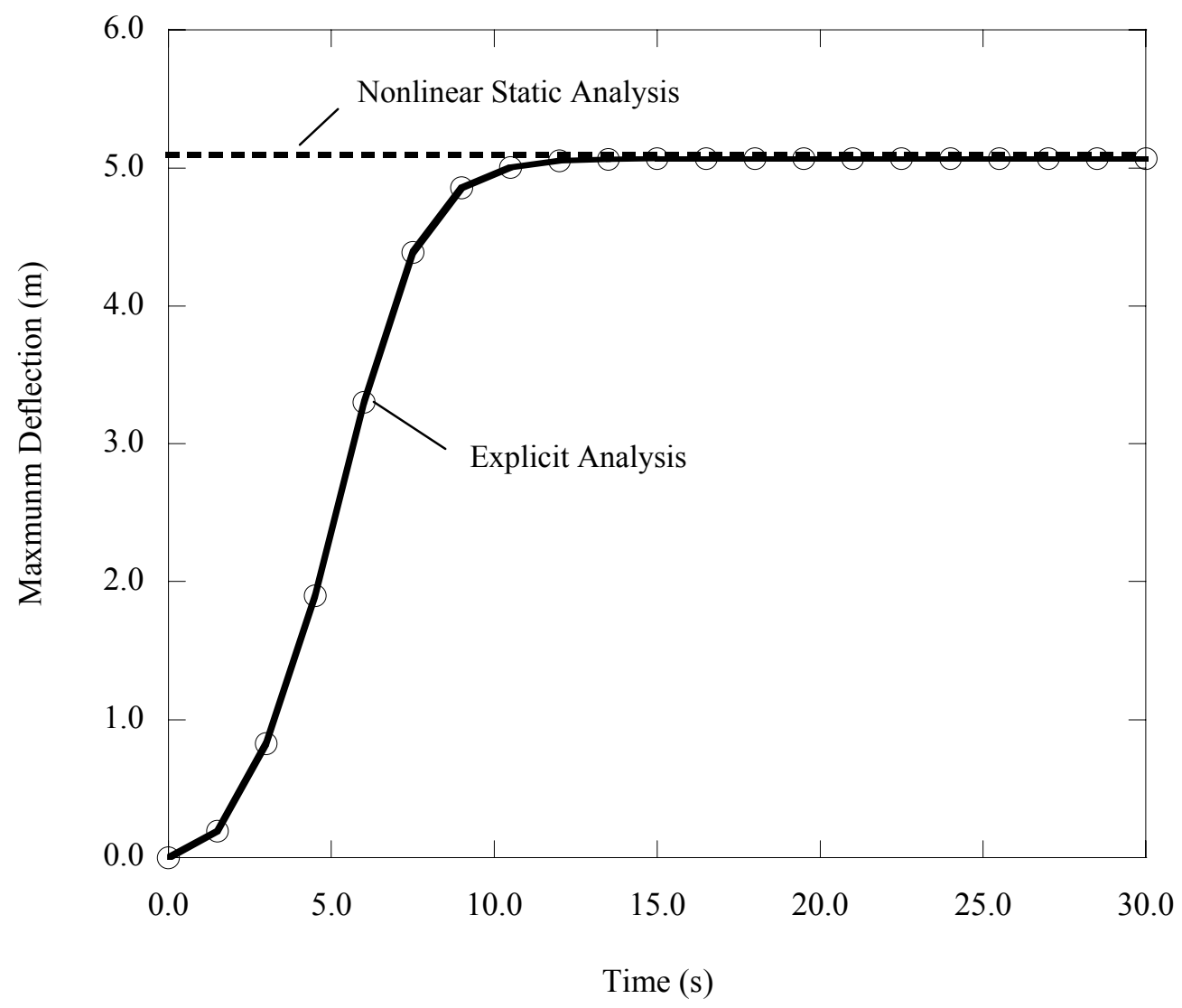

Figure 12: Maximum deflections of solar sail due to solar pressure. 


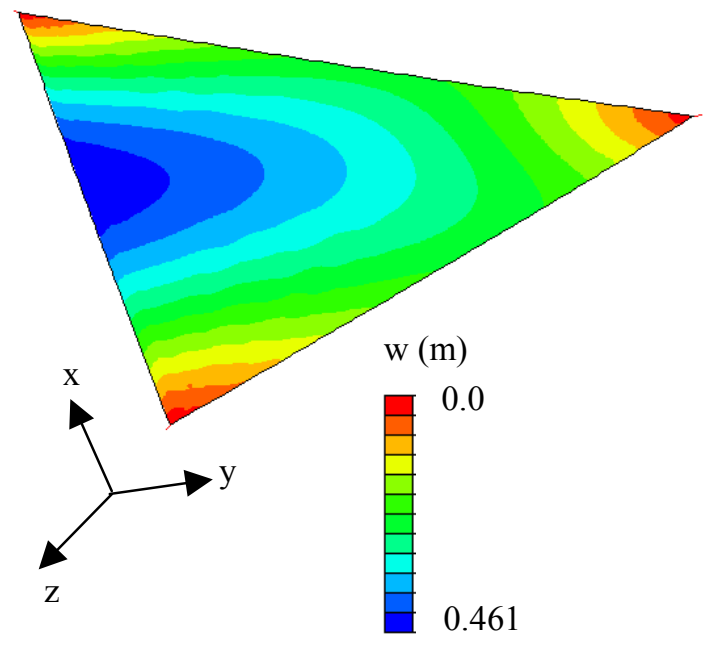

(a) Explicit analysis

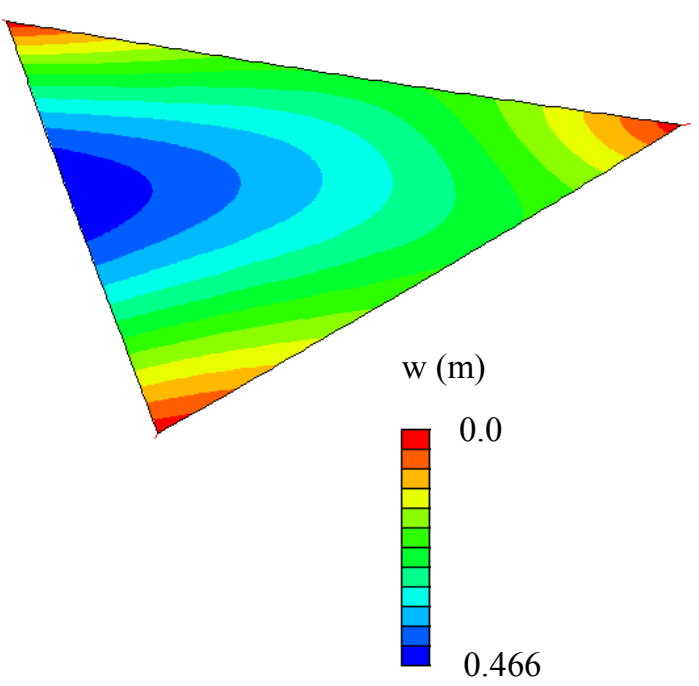

(b) Nonlinear static analysis

Figure 13: Transverse deflection of one membrane quadrant (with attached cables) of a $100 \mathrm{~m}$ solar sail due to the solar pressure.

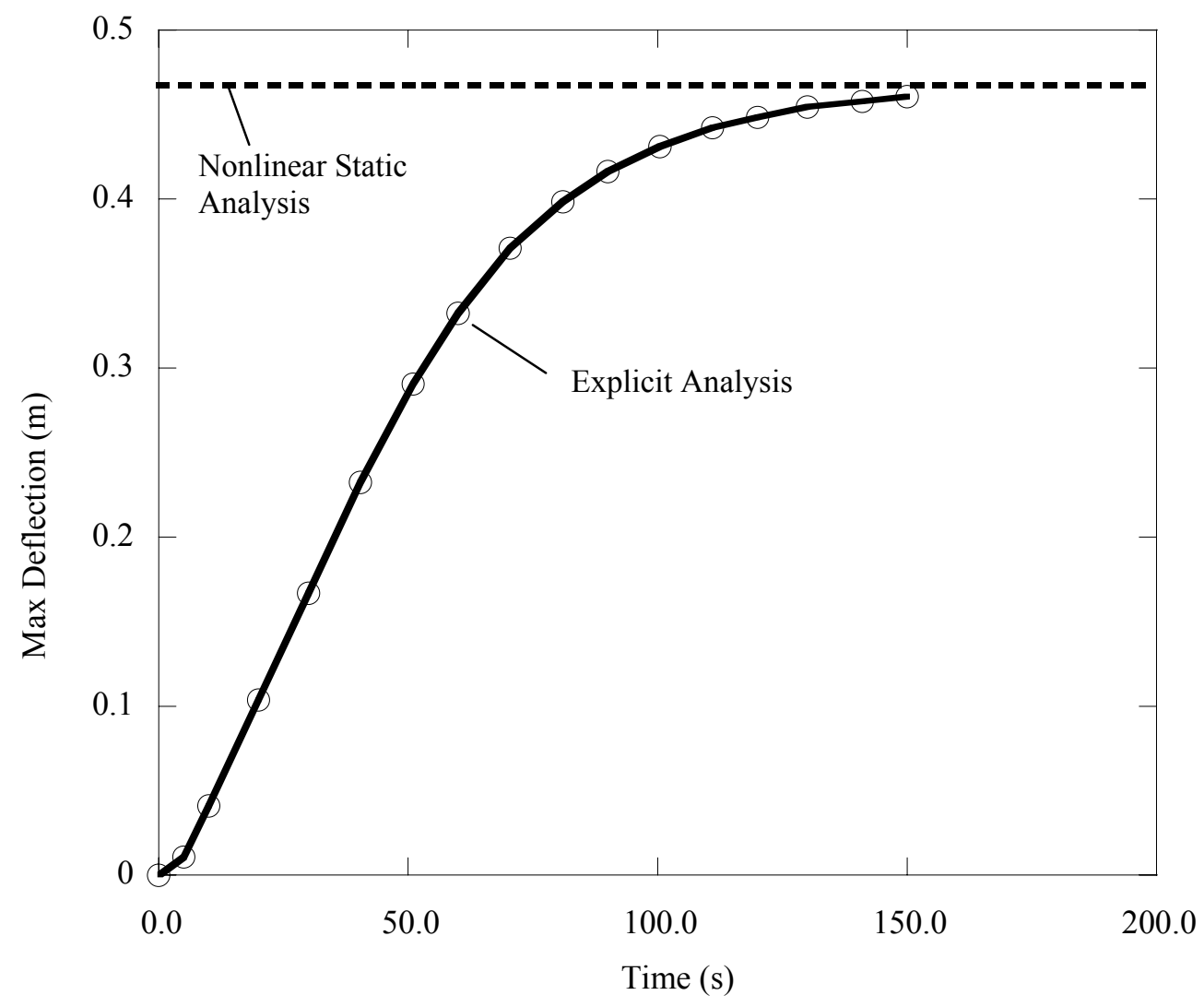

Figure 14: Maximum deflections of solar sail due to solar pressure. 